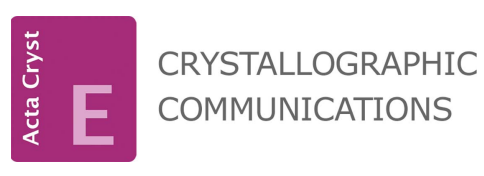

ISSN 2056-9890
Received 11 November 2017

Accepted 5 December 2017

Edited by M. Weil, Vienna University of Technology, Austria

Keywords: crystal structure; titanium; $\eta^{4}$-ketimine complex; half-sandwich complex.

CCDC reference: 1589353

Supporting information: this article has supporting information at journals.iucr.org/e

\section{Crystal structure of the $\eta^{4}$-ketimine titanium complex (diphenylamido- $\kappa N$ ) \{3-methyl-6-[(4- methylphenyl)(phenylazanidyl)methylidene]cyclo- hexa-2,4-dien-1-yl- $\left.\kappa^{2} N, C^{1}\right\}\left(\eta^{5}\right.$-pentamethylcyclo- pentadienyl)titanium(IV)}

\author{
Malte Fischer, Marc Schmidtmann and Rüdiger Beckhaus*
}

Institut für Chemie, Fakultät für Mathematik und Naturwissenschaften, Carl von Ossietzky Universität Oldenburg, 26129 Oldenburg, Germany. *Correspondence e-mail: ruediger.beckhaus@uni-oldenburg.de

The molecular structure of the title titanium(IV) half-sandwich complex, $\left[\mathrm{Ti}\left(\eta^{5}-\mathrm{C}_{10} \mathrm{H}_{15}\right)\left(\eta^{4}-\mathrm{C}_{21} \mathrm{H}_{19} \mathrm{~N}\right)\left(\mathrm{C}_{12} \mathrm{H}_{10} \mathrm{~N}\right)\right]$, shows a three-legged piano-stool geometry at the central $\mathrm{Ti}^{\mathrm{IV}}$ atom, comprising of one pentamethylcyclopentadienyl ligand, one bidentate ketimine ligand in an $\eta^{4}$-coordination mode and one monodentate diphenylamide ligand. Except for van der Waals forces, there are no significant intermolecular interactions in the crystal.

\section{Chemical context}

In the course of our recent investigations with respect to the unusual $\eta^{4}$-coordination mode of the ketimine $\mathrm{PhN}=\mathrm{C}(p$ tolyl) $)_{2}$ ligand in the coordination sphere of titanium (Fischer $e t$ al., 2017; Loose et al., 2014), the bonding situation of the ketimine ligand has been of great interest. This ligand is bonded with the nitrogen atom and one of the ortho-carbon atoms of one para-tolyl moiety to the central titanium(IV) atom, forming five-membered ring structures. Structural details based on the results of X-ray diffraction and of density functional theory calculations at the M06-2X level support the formulation of these complexes as non-classical monoazabutadiene complexes. However, the follow-up chemistry with various multiple bond substrates of the complexes with formulae $\left[\left(\eta^{5}-\mathrm{Cp}^{\#}\right) \mathrm{Ti}\left(\eta^{4}-\mathrm{C}_{21} \mathrm{H}_{19} \mathrm{~N}\right)(\mathrm{Cl})\right]\left(\#=\mathrm{H}_{5}, \mathrm{Me}_{5}\right)$ shows a hidden $\eta^{2}$-imine reactivity to five-membered titanacycles (Fischer et al., 2017), being of high interest due to the importance of $\eta^{2}$-bound imine titanium complexes in industrially relevant hydroaminoalkylation reaction of alkenes (for a recent review on hydroaminoalkylation reactions, see: Chong et al., 2014). In contrast, classical monoazabutadiene complexes (Manssen et al., 2017b; Scholz et al. 1998, 2004) show ring-enlargement reactions to seven-membered titanacycles, using similar substrates (Manssen et al., 2017a; Scholz et al., 1998). Moreover, the ligand framework of the non-classical monoazabutadiene complexes mentioned above is important for their unexpected reactivities. By derivatization of $\left[\left(\eta^{5}-\mathrm{Cp} *\right) \mathrm{Ti}\left(\eta^{4}-\mathrm{C}_{21} \mathrm{H}_{19} \mathrm{~N}\right)(\mathrm{Cl})\right]$ with the dialkyl-substituted lithium amide $\mathrm{LiN}(\mathrm{Me}) \mathrm{Cy}$, the formation of a titanadihydropyrrole is observed as a result of the $1,3-H$-shift in the five-membered ring system in addition to the salt metathesis reaction (Fischer et al., 2017). 
Here we report the synthesis and crystal structure of the title compound $\left(\eta^{5}-\mathrm{C}_{10} \mathrm{H}_{15}\right) \mathrm{Ti}\left(\eta^{4}-\mathrm{C}_{21} \mathrm{H}_{19} \mathrm{~N}\right)\left(\mathrm{C}_{12} \mathrm{H}_{10} \mathrm{~N}\right), \quad \mathbf{1}$, synthesized by the reaction of $\left[\left(\eta^{5}-\mathrm{Cp}^{*}\right) \mathrm{Ti}\left(\eta^{4}-\mathrm{C}_{21} \mathrm{H}_{19} \mathrm{~N}\right)(\mathrm{Cl})\right]$ with the diaryl-substituted lithium amide $\mathrm{LiNPh}_{2}$. Compound 1 maintains the $\eta^{4}$-coordination mode of the ketimine ligand.

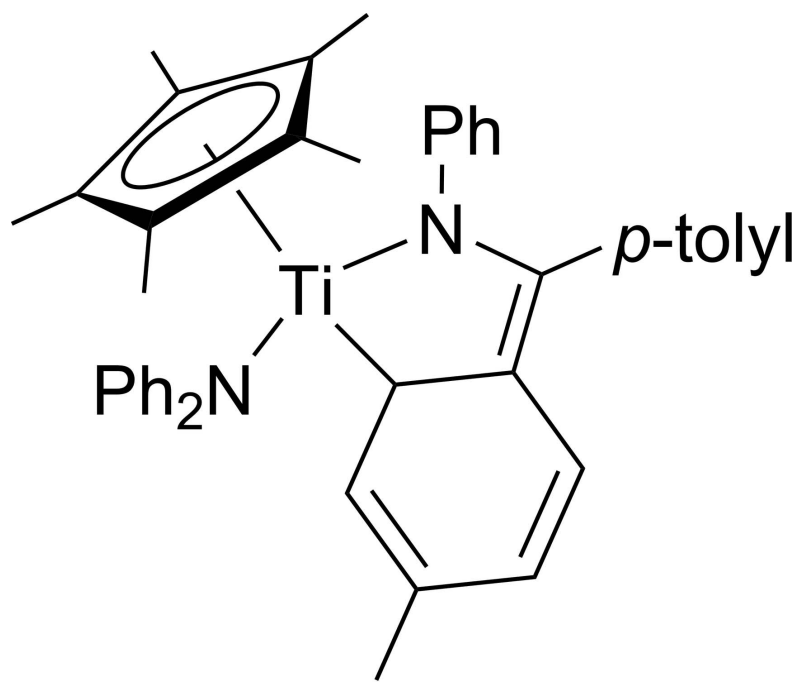

\section{Structural commentary}

Fig. 1 shows the molecular structure of complex 1 for which the $\eta^{4}$-coordination mode of the ketimine ligand is clearly confirmed. The $\mathrm{N} 1-\mathrm{C} 17$ bond length $[1.383$ (3) $\AA]$ is significantly elongated compared to the free ketimine $[1.283$ (1) $\AA$; Loose et al., 2014] and nearly identical to that of the starting complex [( $\left.\left.\eta^{5}-\mathrm{Cp} *\right) \mathrm{Ti}\left(\eta^{4}-\mathrm{C}_{21} \mathrm{H}_{19} \mathrm{~N}\right)(\mathrm{Cl})\right][1.393(2) \AA ;$; (Loose et al., 2014], indicating single-bond character (March, 2007). The C17-C25 bond length [1.414 (4) $\AA]$ is significantly shortened in comparison to the free ketimine [1.497 (1) $\AA$; Loose et al., 2014]. The sum of angles around $\mathrm{C} 17$ [N1-C17-C18 $\left[122.0(2)^{\circ}\right]+\mathrm{N} 1-\mathrm{C} 17-\mathrm{C} 25\left[117.0(2)^{\circ}\right]+\mathrm{C} 18-\mathrm{C} 17-\mathrm{C} 25$ $\left.\left[120.8(2)^{\circ}\right]=359.8^{\circ}\right\}$ indicates $s p^{2}$-hybridization of this atom. Furthermore, localized $\mathrm{C}=\mathrm{C}$ double bonds are found in the $\mathrm{C} 25-\mathrm{C} 30$ aromatic ring $[\mathrm{C} 26-\mathrm{C} 27=1.356(4), \mathrm{C} 28-\mathrm{C} 29=$ 1.355 (4) $\AA$ ] in contrast to the well-balanced $\mathrm{C}$--C distances in the $\mathrm{C} 18-\mathrm{C} 23$ aromatic ring system $(\simeq 1.39 \AA)$. The central titanium(IV) atom is fourfold coordinated in a considerably distorted tetrahedral coordination environment, with N1Ti1-N2 and N1-Ti1-C30 bond angles of 110.42 (9) and $84.23(9)^{\circ}$, respectively. The Ti1-N1 bond length [1.963 (2) $\AA$ ] is shorter than the Ti1-N2 bond length [2.009 (2) $\AA]$ and indicates weak $p_{\pi}-d_{\pi}$ electron donor interactions. The Ti1 $-\mathrm{C} 30$ bond length $[2.259$ (3) $\AA]$ as well as the fold angle of the central five-membered ring system $\left(60.6^{\circ}\right)$ are similar to those in other reported monoazabutadiene complexes (Manssen et al., 2017b; Scholz et al., 1998, 2004). The influence of the $\eta^{4}$-bonding mode of the ketimine ligand can be analysed by the difference $\Delta=[($ Ti1 $-\mathrm{C} 17+$ Ti1 $\mathrm{C} 25) / 2-(\mathrm{Ti} 1-\mathrm{N} 1+\mathrm{Ti} 1-\mathrm{C} 30) / 2]=0.386 \AA($ Scholz et al., 1998). This value is in good agreement with the starting

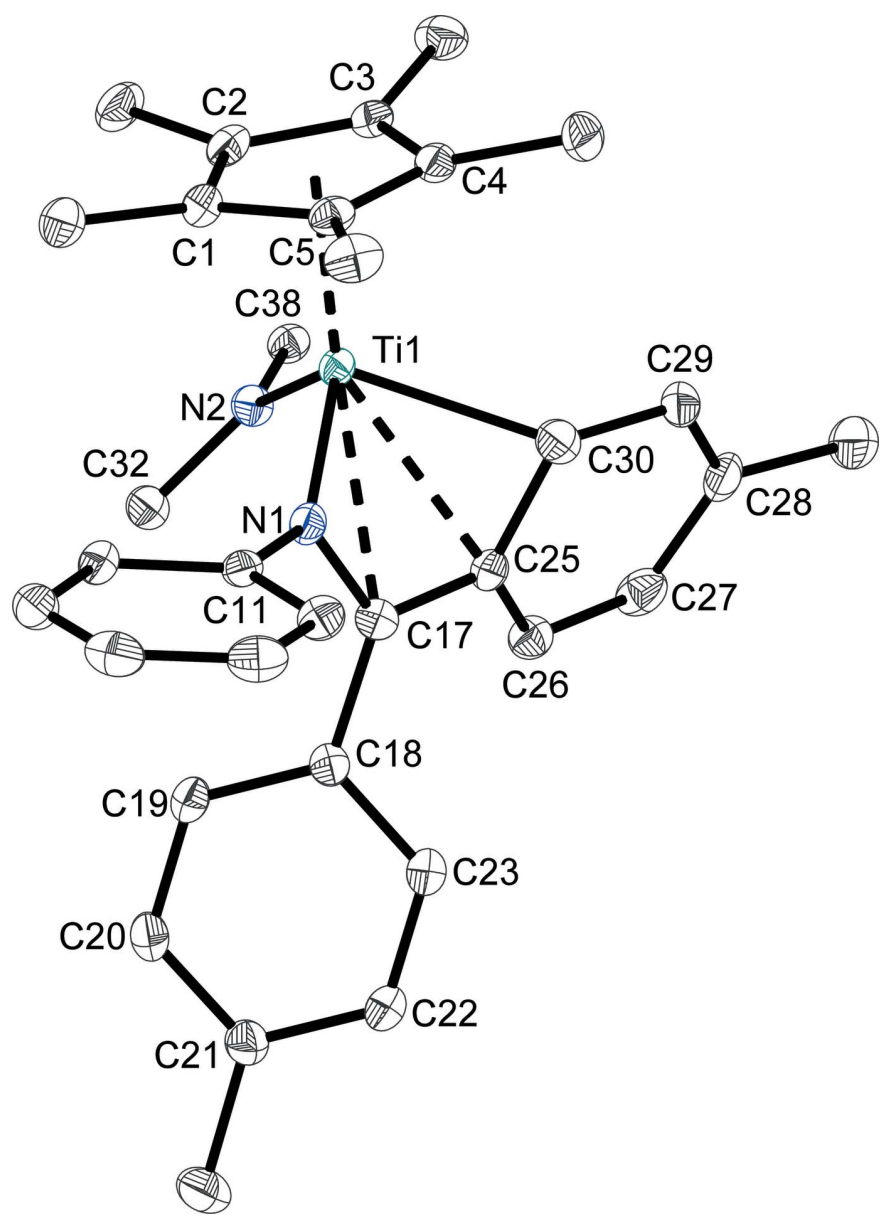

Figure 1

The molecular structure of $\mathbf{1}$, with displacement ellipsoids at the $50 \%$ probability level. $\mathrm{H}$ atoms and phenyl groups of the diphenyl amido moiety have been omitted for clarity.

material (0.326 ̊; Loose et al., 2014) and other related complexes. The terms prone and supine are employed to describe the mode of the monoazadiene orientation in the envelope structure of $\mathbf{1}$, as summarized by Nakamura $e t$ al. (2001). Generally, for monoazabutadiene complexes prone and supine isomers are known. The molecular structure of $\mathbf{1}$ shows the supine isomer.

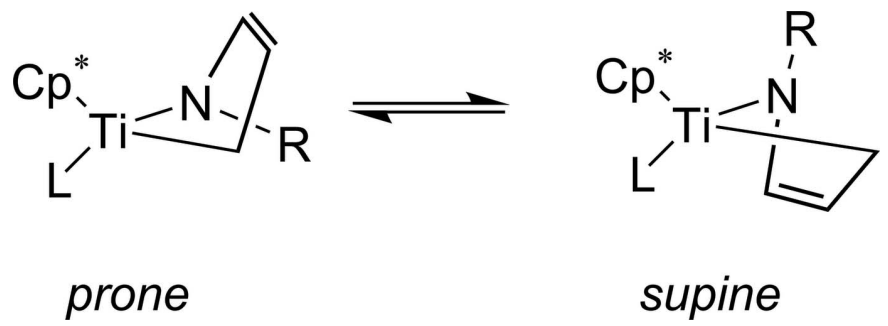

\section{Supramolecular features}

There are no significant supramolecular features in the crystal structure of 1. The crystal packing, shown in Fig. 2, appears to be dominated by van der Waals interactions only. 


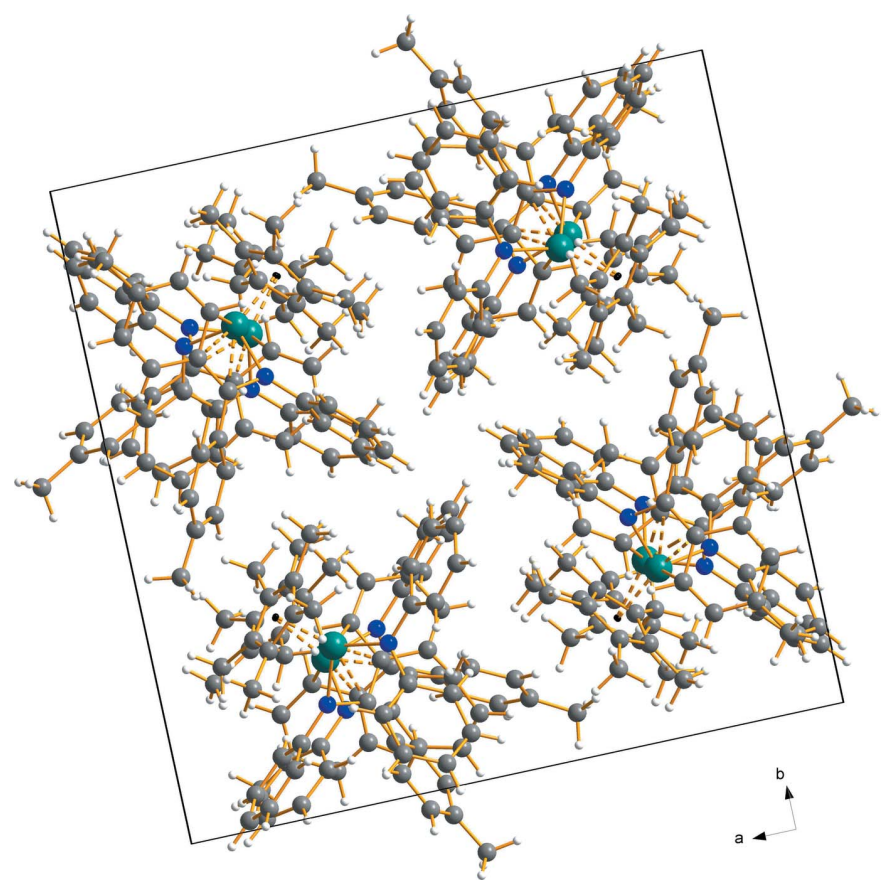

Figure 2

A view along the $c$ axis, showing the packing of the molecules in the crystal structure of complex $\mathbf{1}$. No significant supramolecular features can be observed. Colour code: $\mathrm{C}$ grey, $\mathrm{H}$ colourless, $\mathrm{N}$ blue and Ti turquoise spheres.

\section{Synthesis and crystallization}

All operations were carried out under a dry nitrogen atmosphere using Schlenk techniques or in a glove box. The $\eta^{4}$ ketimine complex $\left[\left(\eta^{5}-\mathrm{Cp} *\right) \mathrm{Ti}\left(\eta^{4}-\mathrm{C}_{21} \mathrm{H}_{19} \mathrm{~N}\right)(\mathrm{Cl})\right]$ and lithium diphenyl amide were prepared according to published procedures (Fischer et al., 2017; Hatakeyama et al., 2012). Solvents were dried according to standard procedures over $\mathrm{Na} / \mathrm{K}$ alloy with benzophenone as indicator and distilled under a nitrogen atmosphere.

$\left[\left(\eta^{5}-\mathrm{Cp}^{*}\right) \mathrm{Ti}\left(\eta^{4}-\mathrm{C}_{21} \mathrm{H}_{19} \mathrm{~N}\right)(\mathrm{Cl})\right] \quad(0.500 \mathrm{~g}, 0.992 \mathrm{mmol})$ and lithium diphenyl amide $(0.174 \mathrm{~g}, 0.992 \mathrm{mmol})$ were dissolved in $12 \mathrm{ml}$ of tetrahydrofuran. After stirring the reaction mixture for $16 \mathrm{~h}$ at room temperature, the solvent was evaporated in a vacuum. The residue was dissolved in $12 \mathrm{ml}$ of toluene, filtered, and the precipitate of $\mathrm{LiCl}$ was washed with toluene $(2 \times 10 \mathrm{ml})$. The combined filtrates were evaporated in a vacuum and the residue was recrystallized from $n$-hexane to yield complex $\mathbf{1}$ as dark-red prisms in $15 \%$ crystalline yield.

\section{Refinement}

Crystal data, data collection and structure refinement details are summarized in Table 1. Hydrogen atoms bonded to carbon atoms, with the exception of $\mathrm{H} 30$ bonded to the ortho-carbon atom that is bonded to titanium, were located from differenceFourier maps but were subsequently fixed in idealized positions using appropriate riding models. Atom H30 was refined freely. The absolute structure was determined (Parsons et al., 2013) by using 3640 quotients.
Table 1

Experimental details.

\begin{tabular}{|c|c|}
\hline \multicolumn{2}{|l|}{ Crystal data } \\
\hline Chemical formula & {$\left[\mathrm{Ti}\left(\mathrm{C}_{10} \mathrm{H}_{15}\right)\left(\mathrm{C}_{21} \mathrm{H}_{19} \mathrm{~N}\right)\left(\mathrm{C}_{12} \mathrm{H}_{10} \mathrm{~N}\right)\right]$} \\
\hline$M_{\mathrm{r}}$ & 636.70 \\
\hline Crystal system, space group & Tetragonal, $P \overline{4} 2_{1} c$ \\
\hline Temperature (K) & 100 \\
\hline$a, c(\AA)$ & $20.0633(4), 16.8156(4)$ \\
\hline$V\left(\AA^{3}\right)$ & $6768.9(3)$ \\
\hline$Z$ & 8 \\
\hline Radiation type & Mo $K \alpha$ \\
\hline$\mu\left(\mathrm{mm}^{-1}\right)$ & 0.29 \\
\hline Crystal size $(\mathrm{mm})$ & $0.40 \times 0.14 \times 0.14$ \\
\hline \multicolumn{2}{|l|}{ Data collection } \\
\hline Diffractometer & Bruker APEXII CCD \\
\hline Absorption correction & $\begin{array}{l}\text { Multi-scan (SADABS; Krause et } \\
\quad \text { al. } 2015)\end{array}$ \\
\hline$T_{\min }, T_{\max }$ & $0.832,1.000$ \\
\hline $\begin{array}{l}\text { No. of measured, independent and } \\
\text { observed }[I>2 \sigma(I)] \text { reflections }\end{array}$ & $152032,9906,8703$ \\
\hline$R_{\text {int }}$ & 0.093 \\
\hline$(\sin \theta / \lambda)_{\max }\left(\AA^{-1}\right)$ & 0.704 \\
\hline \multicolumn{2}{|l|}{ Refinement } \\
\hline$R\left[F^{2}>2 \sigma\left(F^{2}\right)\right], w R\left(F^{2}\right), S$ & $0.043,0.110,1.07$ \\
\hline No. of reflections & 9906 \\
\hline No. of parameters & 426 \\
\hline $\mathrm{H}$-atom treatment & $\begin{array}{l}\mathrm{H} \text { atoms treated by a mixture of } \\
\text { independent and constrained } \\
\text { refinement }\end{array}$ \\
\hline$\Delta \rho_{\max }, \Delta \rho_{\min }\left(\mathrm{e} \AA^{-3}\right)$ & $0.65,-0.53$ \\
\hline Absolute structure & $\begin{array}{l}\text { Flack } x \text { determined using } 3640 \\
\quad \text { quotients }\left[\left(I^{+}\right)-\left(I^{-}\right)\right] /\left[\left(I^{+}\right)+\left(I^{-}\right)\right] \\
\quad \text { (Parsons } \text { et al., 2013) }\end{array}$ \\
\hline Absolute structure parameter & $0.003(8)$ \\
\hline
\end{tabular}

Computer programs: APEX2 and SAINT (Bruker, 2015), SHELXT (Sheldrick, 2015a), SHELXL2014 (Sheldrick, 2015b), DIAMOND (Brandenburg \& Putz, 2006) and publCIF (Westrip, 2010).

\section{References}

Brandenburg, K. \& Putz, H. (2006). DIAMOND. Crystal Impact GbR, Bonn, Germany.

Bruker (2015). APEX2 and SAINT. Bruker AXS Inc., Madison, Wisconsin, USA.

Chong, E., Garcia, P. \& Schafer, L. L. (2014). Synthesis, pp. 305306.

Fischer, M., Schmidtmann, M. \& Beckhaus, R. (2017). Organometallics, doi: 10.1021/acs. organomet. 7b00673.

Hatakeyama, T., Imayoshi, R., Yoshimoto, Y., Ghorai, S. K., Jin, M., Takaya, H., Norisuye, K., Sohrin, Y. \& Nakamura, M. (2012). J. Am. Chem. Soc. 134, 20262-20265.

Krause, L., Herbst-Irmer, R., Sheldrick, G. M. \& Stalke, D. (2015). J. Appl. Cryst. 48, 3-10.

Loose, F., Plettenberg, I., Haase, D., Saak, W., Schmidtmann, M., Schäfer, A., Müller, T. \& Beckhaus, R. (2014). Organometallics, 33, 6785-6795.

Manssen, M., Kahrs, C., Töben, I., Bölte, J.-H., Schmidtmann, M. \& Beckhaus, R. (2017a). Chem. Eur. J. 23, 15827-15833.

Manssen, M., Töben, I., Kahrs, C., Bölte, J.-H., Schmidtmann, M. \& Beckhaus, R. (2017b). Organometallics, 36, 2973-2981.

March, J. (2007). Advanced Organic Chemistry, 6th ed., p. 43. New York: John Wiley \& Sons.

Nakamura, A. \& Mashima, K. (2001). J. Organomet. Chem. 621, 224230.

Parsons, S., Flack, H. D. \& Wagner, T. (2013). Acta Cryst. B69, 249259. 
Scholz, J., Kahlert, S. \& Görls, H. (1998). Organometallics, 17, 28762884.

Scholz, J., Kahlert, S. \& Görls, H. (2004). Organometallics, 23, 1594 1603.
Sheldrick, G. M. (2015a). Acta Cryst. A71, 3-8.

Sheldrick, G. M. (2015b). Acta Cryst. C71, 3-8.

Westrip, S. P. (2010). J. Appl. Cryst. 43, 920-925. 


\section{supporting information}

Acta Cryst. (2018). E74, 34-37 [https://doi.org/10.1107/S2056989017017455]

Crystal structure of the $\eta^{4}$-ketimine titanium complex (diphenylamido- $\left.k \mathrm{~N}\right)\{3$ methyl-6-[(4-methylphenyl)(phenylazanidyl)methylidene]cyclohexa-2,4-dien-1yl- $\left.\kappa^{2} N, C^{1}\right\}\left(\eta^{5}\right.$-pentamethylcyclopentadienyl)titanium(IV)

\section{Malte Fischer, Marc Schmidtmann and Rüdiger Beckhaus}

Computing details

Data collection: APEX2 (Bruker, 2015); cell refinement: SAINT (Bruker, 2015); data reduction: SAINT (Bruker, 2015); program(s) used to solve structure: SHELXT (Sheldrick, 2015a); program(s) used to refine structure: SHELXL2014 (Sheldrick, 2015b); molecular graphics: DIAMOND (Brandenburg \& Putz, 2006); software used to prepare material for publication: publCIF (Westrip, 2010).

(Diphenylamido- $\kappa N)\left\{3\right.$-methyl-6-[(4-methylphenyl) (phenylazanidyl)methylidene]cyclohexa-2,4-dien-1-yl- $\left.\kappa^{2} N, C^{1}\right\}$ ( $\eta^{5}$-pentamethylcyclopentadienyl)titanium(IV)

Crystal data

$\left[\mathrm{Ti}\left(\mathrm{C}_{10} \mathrm{H}_{15}\right)\left(\mathrm{C}_{21} \mathrm{H}_{19} \mathrm{~N}\right)\left(\mathrm{C}_{12} \mathrm{H}_{10} \mathrm{~N}\right)\right]$

$M_{r}=636.70$

Tetragonal, $P \overline{4}{ }_{1} c$

$a=20.0633$ (4) $\AA$

$c=16.8156(4) \AA$

$V=6768.9(3) \AA^{3}$

$Z=8$

$F(000)=2704$

\section{Data collection}

Bruker APEXII CCD

diffractometer

Radiation source: sealed tube $\varphi$ and $\omega$ scans

Absorption correction: multi-scan

(SADABS; Krause et al., 2015)

$T_{\min }=0.832, T_{\max }=1.000$

152032 measured reflections

Refinement

Refinement on $F^{2}$

Least-squares matrix: full

$R\left[F^{2}>2 \sigma\left(F^{2}\right)\right]=0.043$

$w R\left(F^{2}\right)=0.110$

$S=1.07$

9906 reflections

426 parameters

0 restraints
$D_{\mathrm{x}}=1.250 \mathrm{Mg} \mathrm{m}^{-3}$

Mo $K \alpha$ radiation, $\lambda=0.71073 \AA$

Cell parameters from 9899 reflections

$\theta=2.3-27.7^{\circ}$

$\mu=0.29 \mathrm{~mm}^{-1}$

$T=100 \mathrm{~K}$

Tetragonal prism, dark red

$0.40 \times 0.14 \times 0.14 \mathrm{~mm}$

9906 independent reflections

8703 reflections with $I>2 \sigma(I)$

$R_{\text {int }}=0.093$

$\theta_{\text {max }}=30.0^{\circ}, \theta_{\min }=1.4^{\circ}$

$h=-28 \rightarrow 28$

$k=-28 \rightarrow 28$

$l=-23 \rightarrow 23$

Primary atom site location: structure-invariant direct methods

Secondary atom site location: difference Fourier map

Hydrogen site location: difference Fourier map

$\mathrm{H}$ atoms treated by a mixture of independent and constrained refinement 
$w=1 /\left[\sigma^{2}\left(F_{\mathrm{o}}^{2}\right)+(0.060 P)^{2}+2 . P\right]$

where $P=\left(F_{\mathrm{o}}^{2}+2 F_{\mathrm{c}}^{2}\right) / 3$

$(\Delta / \sigma)_{\max }=0.001$

$\Delta \rho_{\max }=0.65$ e $\AA^{-3}$

$\Delta \rho_{\min }=-0.53$ e $\AA^{-3}$

Special details

Geometry. All esds (except the esd in the dihedral angle between two 1.s. planes) are estimated using the full covariance matrix. The cell esds are taken into account individually in the estimation of esds in distances, angles and torsion angles; correlations between esds in cell parameters are only used when they are defined by crystal symmetry. An approximate (isotropic) treatment of cell esds is used for estimating esds involving l.s. planes.

Fractional atomic coordinates and isotropic or equivalent isotropic displacement parameters $\left(\hat{A}^{2}\right)$

\begin{tabular}{|c|c|c|c|c|}
\hline & $x$ & $y$ & $z$ & $U_{\text {iso }} * / U_{\text {eq }}$ \\
\hline Ti1 & $0.25521(2)$ & $0.77115(2)$ & $0.52922(3)$ & $0.01349(10)$ \\
\hline N1 & $0.33928(10)$ & $0.74376(11)$ & $0.57924(12)$ & $0.0165(4)$ \\
\hline N2 & $0.26913(11)$ & $0.85300(10)$ & $0.46239(13)$ & $0.0165(4)$ \\
\hline $\mathrm{C} 1$ & $0.24245(13)$ & $0.67486(13)$ & $0.44812(15)$ & $0.0183(5)$ \\
\hline $\mathrm{C} 2$ & $0.19622(13)$ & $0.72333(13)$ & $0.41991(16)$ & $0.0191(5)$ \\
\hline $\mathrm{C} 3$ & $0.14749(12)$ & $0.73412(12)$ & $0.47990(15)$ & $0.0173(5)$ \\
\hline $\mathrm{C} 4$ & $0.16372(13)$ & $0.69257(13)$ & $0.54563(15)$ & $0.0175(5)$ \\
\hline $\mathrm{C} 5$ & $0.22181(12)$ & $0.65600(12)$ & $0.52647(16)$ & $0.0178(5)$ \\
\hline C6 & $0.29283(15)$ & $0.63923(15)$ & $0.39780(19)$ & $0.0265(6)$ \\
\hline H6A & 0.3143 & 0.6711 & 0.3619 & $0.040^{*}$ \\
\hline H6B & 0.2705 & 0.6046 & 0.3665 & $0.040^{*}$ \\
\hline $\mathrm{H} 6 \mathrm{C}$ & 0.3266 & 0.6187 & 0.4321 & $0.040^{*}$ \\
\hline $\mathrm{C} 7$ & $0.19600(15)$ & $0.75307(16)$ & $0.33785(16)$ & $0.0257(6)$ \\
\hline H7A & 0.1748 & 0.7971 & 0.3395 & $0.039^{*}$ \\
\hline $\mathrm{H} 7 \mathrm{~B}$ & 0.1711 & 0.7239 & 0.3018 & $0.039^{*}$ \\
\hline $\mathrm{H} 7 \mathrm{C}$ & 0.2420 & 0.7576 & 0.3189 & $0.039^{*}$ \\
\hline $\mathrm{C} 8$ & $0.08424(13)$ & $0.77324(14)$ & $0.47078(19)$ & $0.0244(5)$ \\
\hline H8A & 0.0732 & 0.7948 & 0.5214 & $0.037^{*}$ \\
\hline H8B & 0.0480 & 0.7432 & 0.4554 & $0.037^{*}$ \\
\hline $\mathrm{H} 8 \mathrm{C}$ & 0.0902 & 0.8073 & 0.4296 & $0.037^{*}$ \\
\hline C9 & $0.12056(15)$ & $0.68160(15)$ & $0.61720(18)$ & $0.0249(6)$ \\
\hline H9A & 0.1486 & 0.6780 & 0.6647 & $0.037^{*}$ \\
\hline H9B & 0.0950 & 0.6404 & 0.6104 & $0.037^{*}$ \\
\hline $\mathrm{H} 9 \mathrm{C}$ & 0.0899 & 0.7192 & 0.6233 & $0.037^{*}$ \\
\hline $\mathrm{C} 10$ & $0.25101(16)$ & 0.60119 (14) & $0.57619(18)$ & $0.0255(6)$ \\
\hline $\mathrm{H} 10 \mathrm{~A}$ & 0.2971 & 0.5929 & 0.5595 & $0.038^{*}$ \\
\hline H10B & 0.2246 & 0.5605 & 0.5693 & $0.038^{*}$ \\
\hline $\mathrm{H} 10 \mathrm{C}$ & 0.2504 & 0.6144 & 0.6323 & $0.038^{*}$ \\
\hline $\mathrm{C} 11$ & $0.38873(12)$ & $0.69317(13)$ & $0.58313(16)$ & $0.0160(5)$ \\
\hline $\mathrm{C} 12$ & $0.40136(14)$ & $0.66049(14)$ & $0.65474(17)$ & $0.0212(5)$ \\
\hline H12 & 0.3775 & 0.6728 & 0.7013 & $0.025^{*}$ \\
\hline $\mathrm{C} 13$ & $0.44865(15)$ & $0.61008(15)$ & $0.65820(19)$ & $0.0255(6)$ \\
\hline H13 & 0.4564 & 0.5873 & 0.7068 & $0.031^{*}$ \\
\hline C14 & $0.48470(14)$ & $0.59293(14)$ & $0.5909(2)$ & $0.0259(6)$ \\
\hline
\end{tabular}

Absolute structure: Flack $x$ determined using 3640 quotients $\left[\left(I^{+}\right)-\left(I^{-}\right)\right] /\left[\left(I^{+}\right)+\left(I^{-}\right)\right]$(Parsons et al., 2013)

Absolute structure parameter: $0.003(8)$ 


\begin{tabular}{|c|c|c|c|c|}
\hline H14 & 0.5173 & 0.5587 & 0.5934 & $0.031 *$ \\
\hline $\mathrm{C} 15$ & $0.47305(14)$ & $0.62589(14)$ & $0.51979(19)$ & $0.0236(6)$ \\
\hline H15 & 0.4982 & 0.6146 & 0.4739 & $0.028^{*}$ \\
\hline $\mathrm{C} 16$ & $0.42487(13)$ & $0.67536(13)$ & $0.51536(16)$ & $0.0196(5)$ \\
\hline H16 & 0.4164 & 0.6971 & 0.4662 & $0.024^{*}$ \\
\hline $\mathrm{C} 17$ & $0.34882(13)$ & $0.80236(13)$ & $0.62103(16)$ & $0.0184(5)$ \\
\hline $\mathrm{C} 18$ & $0.41617(13)$ & $0.83181(13)$ & $0.63272(16)$ & $0.0167(5)$ \\
\hline C19 & $0.46455(13)$ & $0.83115(13)$ & $0.57330(15)$ & $0.0188(5)$ \\
\hline H19 & 0.4554 & 0.8096 & 0.5242 & $0.023^{*}$ \\
\hline $\mathrm{C} 20$ & $0.52607(14)$ & $0.86168(14)$ & $0.58502(17)$ & $0.0216(5)$ \\
\hline $\mathrm{H} 20$ & 0.5584 & 0.8606 & 0.5437 & $0.026^{*}$ \\
\hline $\mathrm{C} 21$ & $0.54113(14)$ & $0.89386(14)$ & $0.65630(17)$ & $0.0214(5)$ \\
\hline $\mathrm{C} 22$ & $0.49367(14)$ & $0.89274(15)$ & $0.71652(17)$ & $0.0227(6)$ \\
\hline $\mathrm{H} 22$ & 0.5033 & 0.9132 & 0.7661 & $0.027^{*}$ \\
\hline $\mathrm{C} 23$ & $0.43237(14)$ & $0.86209(14)$ & $0.70517(17)$ & $0.0207(5)$ \\
\hline $\mathrm{H} 23$ & 0.4009 & 0.8617 & 0.7473 & $0.025^{*}$ \\
\hline $\mathrm{C} 24$ & $0.60631(16)$ & $0.93054(18)$ & $0.6678(2)$ & $0.0332(7)$ \\
\hline $\mathrm{H} 24 \mathrm{~A}$ & 0.6385 & 0.9155 & 0.6278 & $0.050^{*}$ \\
\hline H24B & 0.6237 & 0.9213 & 0.7211 & $0.050^{*}$ \\
\hline $\mathrm{H} 24 \mathrm{C}$ & 0.5989 & 0.9786 & 0.6618 & $0.050^{*}$ \\
\hline $\mathrm{C} 25$ & $0.29109(13)$ & $0.83635(13)$ & $0.64712(15)$ & $0.0166(5)$ \\
\hline $\mathrm{C} 26$ & $0.29111(14)$ & $0.90781(13)$ & $0.65934(16)$ & $0.0194(5)$ \\
\hline $\mathrm{H} 26$ & 0.3302 & 0.9327 & 0.6480 & $0.023^{*}$ \\
\hline $\mathrm{C} 27$ & $0.23609(15)$ & $0.93964(14)$ & $0.68677(17)$ & $0.0236(6)$ \\
\hline $\mathrm{H} 27$ & 0.2377 & 0.9866 & 0.6940 & $0.028^{*}$ \\
\hline $\mathrm{C} 28$ & $0.17566(14)$ & 0.90498 (16) & $0.70515(17)$ & $0.0238(6)$ \\
\hline $\mathrm{C} 29$ & $0.17200(14)$ & $0.83910(16)$ & $0.68804(16)$ & $0.0220(5)$ \\
\hline $\mathrm{H} 29$ & 0.1320 & 0.8156 & 0.6993 & $0.026^{*}$ \\
\hline $\mathrm{C} 30$ & $0.22712(13)$ & $0.80375(14)$ & $0.65334(17)$ & $0.0203(5)$ \\
\hline H30 & $0.2255(16)$ & $0.7539(16)$ & $0.6633(19)$ & $0.018(8)^{*}$ \\
\hline $\mathrm{C} 31$ & $0.11923(16)$ & $0.94261(18)$ & $0.7438(2)$ & $0.0341(7)$ \\
\hline $\mathrm{H} 31 \mathrm{~A}$ & 0.1344 & 0.9613 & 0.7945 & $0.051 *$ \\
\hline H31B & 0.0819 & 0.9122 & 0.7534 & $0.051 *$ \\
\hline $\mathrm{H} 31 \mathrm{C}$ & 0.1047 & 0.9788 & 0.7086 & $0.051 *$ \\
\hline $\mathrm{C} 32$ & $0.33448(13)$ & $0.86856(13)$ & $0.43620(16)$ & $0.0181(5)$ \\
\hline $\mathrm{C} 33$ & $0.37170(14)$ & $0.82304(15)$ & $0.39128(15)$ & $0.0206(5)$ \\
\hline $\mathrm{H} 33$ & 0.3531 & 0.7811 & 0.3774 & $0.025^{*}$ \\
\hline C34 & $0.43614(14)$ & $0.83944(17)$ & $0.36691(17)$ & $0.0259(6)$ \\
\hline H34 & 0.4613 & 0.8082 & 0.3368 & $0.031^{*}$ \\
\hline C35 & $0.46377(15)$ & 0.90029 (17) & $0.38585(19)$ & $0.0284(6)$ \\
\hline H35 & 0.5076 & 0.9112 & 0.3687 & $0.034 *$ \\
\hline C36 & $0.42683(15)$ & $0.94556(16)$ & $0.4303(2)$ & $0.0280(6)$ \\
\hline H36 & 0.4457 & 0.9875 & 0.4438 & $0.034 *$ \\
\hline $\mathrm{C} 37$ & $0.36303(14)$ & $0.93018(14)$ & $0.45505(18)$ & $0.0229(6)$ \\
\hline H37 & 0.3383 & 0.9617 & 0.4851 & $0.028^{*}$ \\
\hline $\mathrm{C} 38$ & $0.22008(13)$ & $0.89928(12)$ & $0.43968(15)$ & $0.0159(5)$ \\
\hline C39 & 0.16483 (13) & $0.91041(13)$ & $0.48870(16)$ & $0.0192(5)$ \\
\hline H39 & 0.1619 & 0.8885 & 0.5386 & $0.023 *$ \\
\hline
\end{tabular}




$\begin{array}{lllll}\text { C40 } & 0.11412(14) & 0.95335(14) & 0.46488(19) & 0.0234(5) \\ \text { H40 } & 0.0763 & 0.9595 & 0.4981 & 0.028^{*} \\ \text { C41 } & 0.11815(15) & 0.98732(15) & 0.39313(18) & 0.0244(6) \\ \text { H41 } & 0.0833 & 1.0164 & 0.3770 & 0.029^{*} \\ \text { C42 } & 0.17374(15) & 0.97823(14) & 0.34527(17) & 0.0229(5) \\ \text { H42 } & 0.1774 & 1.0023 & 0.2968 & 0.027^{*} \\ \text { C43 } & 0.22390(14) & 0.93457(13) & 0.36723(16) & 0.0198(5) \\ \text { H43 } & 0.2612 & 0.9283 & 0.3332 & 0.024^{*}\end{array}$

Atomic displacement parameters $\left(\AA^{2}\right)$

\begin{tabular}{|c|c|c|c|c|c|c|}
\hline & $U^{11}$ & $U^{22}$ & $U^{33}$ & $U^{12}$ & $U^{13}$ & $U^{23}$ \\
\hline Til & $0.0154(2)$ & $0.01400(19)$ & 0.01109 (17) & $-0.00003(14)$ & $-0.00026(16)$ & $0.00082(16)$ \\
\hline N1 & $0.0164(9)$ & $0.0204(10)$ & $0.0127(9)$ & $0.0021(8)$ & $0.0002(8)$ & $-0.0009(8)$ \\
\hline N2 & $0.0169(9)$ & $0.0170(9)$ & $0.0156(10)$ & $-0.0011(8)$ & $-0.0004(8)$ & $0.0026(8)$ \\
\hline $\mathrm{C} 1$ & $0.0196(12)$ & $0.0190(11)$ & $0.0165(11)$ & $-0.0002(9)$ & $0.0006(9)$ & $-0.0032(9)$ \\
\hline $\mathrm{C} 2$ & $0.0232(12)$ & $0.0206(12)$ & $0.0135(11)$ & $-0.0013(10)$ & $-0.0028(10)$ & $-0.0017(10)$ \\
\hline $\mathrm{C} 3$ & $0.0177(11)$ & $0.0177(11)$ & $0.0164(11)$ & $-0.0027(9)$ & $-0.0017(9)$ & $-0.0027(9)$ \\
\hline $\mathrm{C} 4$ & $0.0192(11)$ & $0.0173(11)$ & $0.0160(12)$ & $-0.0052(9)$ & $0.0008(9)$ & $-0.0005(9)$ \\
\hline $\mathrm{C} 5$ & $0.0205(11)$ & $0.0149(10)$ & $0.0179(11)$ & $-0.0033(9)$ & $-0.0027(10)$ & $-0.0004(10)$ \\
\hline C6 & $0.0247(14)$ & $0.0273(14)$ & $0.0274(15)$ & $0.0012(11)$ & $0.0028(11)$ & $-0.0112(12)$ \\
\hline $\mathrm{C} 7$ & $0.0323(15)$ & $0.0313(15)$ & $0.0136(12)$ & $-0.0039(12)$ & $-0.0032(11)$ & $0.0005(11)$ \\
\hline $\mathrm{C} 8$ & $0.0197(12)$ & $0.0237(12)$ & $0.0296(14)$ & $0.0013(10)$ & $-0.0030(12)$ & $-0.0043(12)$ \\
\hline C9 & $0.0267(14)$ & $0.0287(14)$ & $0.0194(13)$ & $-0.0088(11)$ & $0.0063(11)$ & $-0.0003(11)$ \\
\hline $\mathrm{C} 10$ & $0.0305(14)$ & $0.0181(12)$ & $0.0279(14)$ & $-0.0017(11)$ & $-0.0062(12)$ & $0.0037(11)$ \\
\hline $\mathrm{C} 11$ & $0.0151(11)$ & $0.0166(11)$ & $0.0161(11)$ & $-0.0006(9)$ & $-0.0016(9)$ & $-0.0023(9)$ \\
\hline $\mathrm{C} 12$ & $0.0219(13)$ & $0.0221(13)$ & $0.0196(12)$ & $0.0003(10)$ & $-0.0024(10)$ & $0.0001(10)$ \\
\hline $\mathrm{C} 13$ & $0.0254(14)$ & $0.0212(13)$ & $0.0300(15)$ & $0.0004(11)$ & $-0.0085(12)$ & $0.0039(11)$ \\
\hline $\mathrm{C} 14$ & $0.0198(13)$ & $0.0180(12)$ & $0.0399(17)$ & $0.0018(10)$ & $-0.0038(12)$ & $-0.0043(12)$ \\
\hline $\mathrm{C} 15$ & $0.0212(12)$ & $0.0221(12)$ & $0.0276(15)$ & $-0.0021(10)$ & $0.0033(11)$ & $-0.0081(11)$ \\
\hline $\mathrm{C} 16$ & $0.0204(12)$ & $0.0218(12)$ & $0.0167(13)$ & $0.0004(9)$ & $0.0004(10)$ & $-0.0040(10)$ \\
\hline $\mathrm{C} 17$ & $0.0166(11)$ & $0.0199(12)$ & $0.0188(12)$ & $-0.0003(9)$ & $-0.0004(10)$ & $-0.0005(10)$ \\
\hline $\mathrm{C} 18$ & $0.0168(11)$ & $0.0162(11)$ & $0.0170(12)$ & $0.0001(9)$ & $0.0013(9)$ & $0.0000(9)$ \\
\hline C19 & $0.0219(12)$ & $0.0215(12)$ & $0.0131(11)$ & $0.0005(10)$ & $0.0007(10)$ & $-0.0008(10)$ \\
\hline $\mathrm{C} 20$ & $0.0206(12)$ & $0.0253(13)$ & $0.0188(12)$ & $-0.0009(10)$ & $0.0056(10)$ & $-0.0014(11)$ \\
\hline $\mathrm{C} 21$ & $0.0190(12)$ & $0.0220(12)$ & $0.0231(13)$ & $-0.0008(10)$ & $0.0026(11)$ & $-0.0042(11)$ \\
\hline $\mathrm{C} 22$ & $0.0227(13)$ & $0.0279(14)$ & $0.0176(12)$ & $-0.0010(11)$ & $0.0009(11)$ & $-0.0067(11)$ \\
\hline $\mathrm{C} 23$ & $0.0206(13)$ & $0.0238(13)$ & $0.0175(13)$ & $0.0003(10)$ & $0.0026(10)$ & $-0.0028(10)$ \\
\hline $\mathrm{C} 24$ & $0.0207(14)$ & $0.0389(18)$ & $0.0401(19)$ & $-0.0081(12)$ & $0.0040(13)$ & $-0.0120(15)$ \\
\hline $\mathrm{C} 25$ & $0.0182(11)$ & $0.0198(12)$ & $0.0120(11)$ & $-0.0008(9)$ & $0.0000(9)$ & $0.0007(9)$ \\
\hline $\mathrm{C} 26$ & $0.0208(12)$ & $0.0193(12)$ & $0.0179(12)$ & $-0.0009(9)$ & $-0.0010(10)$ & $0.0001(10)$ \\
\hline $\mathrm{C} 27$ & $0.0260(14)$ & $0.0214(12)$ & $0.0235(13)$ & $0.0049(11)$ & $-0.0050(11)$ & $-0.0060(10)$ \\
\hline $\mathrm{C} 28$ & $0.0216(13)$ & $0.0356(16)$ & $0.0142(12)$ & $0.0077(11)$ & $-0.0013(10)$ & $-0.0070(11)$ \\
\hline $\mathrm{C} 29$ & $0.0173(12)$ & $0.0330(15)$ & $0.0156(12)$ & $0.0003(10)$ & $0.0015(10)$ & $-0.0011(11)$ \\
\hline $\mathrm{C} 30$ & $0.0185(11)$ & $0.0215(12)$ & $0.0210(13)$ & $-0.0004(10)$ & $0.0003(10)$ & $0.0007(10)$ \\
\hline $\mathrm{C} 31$ & $0.0248(15)$ & $0.0434(19)$ & $0.0342(17)$ & $0.0030(13)$ & $0.0025(13)$ & $-0.0174(15)$ \\
\hline C32 & $0.0177(12)$ & $0.0214(12)$ & $0.0154(12)$ & $0.0002(9)$ & $-0.0008(9)$ & $0.0051(10)$ \\
\hline C33 & $0.0231(13)$ & $0.0270(13)$ & $0.0119(11)$ & $0.0014(10)$ & $-0.0018(10)$ & $0.0019(10)$ \\
\hline
\end{tabular}




\begin{tabular}{lllllll}
\hline C34 & $0.0209(13)$ & $0.0418(17)$ & $0.0150(12)$ & $0.0050(12)$ & $0.0000(10)$ & $0.0056(12)$ \\
C35 & $0.0194(13)$ & $0.0415(17)$ & $0.0243(14)$ & $-0.0005(12)$ & $0.0009(11)$ & $0.0130(13)$ \\
C36 & $0.0224(13)$ & $0.0272(15)$ & $0.0345(17)$ & $-0.0062(11)$ & $-0.0035(12)$ & $0.0108(13)$ \\
C37 & $0.0208(12)$ & $0.0207(12)$ & $0.0272(15)$ & $0.0009(10)$ & $-0.0002(11)$ & $0.0029(11)$ \\
C38 & $0.0180(11)$ & $0.0143(10)$ & $0.0154(11)$ & $-0.0016(9)$ & $-0.0013(9)$ & $0.0003(9)$ \\
C39 & $0.0205(12)$ & $0.0179(12)$ & $0.0192(13)$ & $-0.0010(9)$ & $0.0029(10)$ & $0.0034(9)$ \\
C40 & $0.0212(12)$ & $0.0230(12)$ & $0.0259(14)$ & $0.0009(10)$ & $0.0029(11)$ & $0.0020(12)$ \\
C41 & $0.0234(13)$ & $0.0237(13)$ & $0.0262(14)$ & $0.0048(11)$ & $-0.0050(11)$ & $0.0014(11)$ \\
C42 & $0.0302(14)$ & $0.0221(13)$ & $0.0164(12)$ & $0.0018(11)$ & $-0.0020(11)$ & $0.0018(10)$ \\
C43 & $0.0239(13)$ & $0.0203(12)$ & $0.0152(12)$ & $0.0008(10)$ & $0.0005(10)$ & $0.0008(9)$ \\
\hline
\end{tabular}

Geometric parameters $\left(A,{ }^{\circ}\right)$

\begin{tabular}{|c|c|c|c|}
\hline $\mathrm{Ti} 1-\mathrm{N} 1$ & $1.963(2)$ & $\mathrm{C} 17-\mathrm{C} 18$ & $1.488(4)$ \\
\hline Ti1-N2 & $2.009(2)$ & $\mathrm{C} 18-\mathrm{C} 19$ & $1.393(4)$ \\
\hline Ti1 $-\mathrm{C} 30$ & $2.259(3)$ & $\mathrm{C} 18-\mathrm{C} 23$ & $1.400(4)$ \\
\hline $\mathrm{Ti} 1-\mathrm{C} 1$ & $2.379(3)$ & $\mathrm{C} 19-\mathrm{C} 20$ & $1.392(4)$ \\
\hline $\mathrm{Ti} 1-\mathrm{C} 2$ & $2.387(3)$ & C19-H19 & 0.9500 \\
\hline $\mathrm{Ti} 1-\mathrm{C} 5$ & $2.406(2)$ & $\mathrm{C} 20-\mathrm{C} 21$ & $1.394(4)$ \\
\hline $\mathrm{Ti} 1-\mathrm{C} 3$ & $2.431(2)$ & $\mathrm{C} 20-\mathrm{H} 20$ & 0.9500 \\
\hline Til-C4 & $2.435(3)$ & $\mathrm{C} 21-\mathrm{C} 22$ & $1.390(4)$ \\
\hline Ti1 $-\mathrm{C} 25$ & $2.482(3)$ & $\mathrm{C} 21-\mathrm{C} 24$ & $1.513(4)$ \\
\hline Ti1 $-\mathrm{C} 17$ & $2.511(3)$ & $\mathrm{C} 22-\mathrm{C} 23$ & $1.388(4)$ \\
\hline $\mathrm{N} 1-\mathrm{C} 17$ & $1.383(3)$ & $\mathrm{C} 22-\mathrm{H} 22$ & 0.9500 \\
\hline $\mathrm{N} 1-\mathrm{C} 11$ & $1.421(3)$ & $\mathrm{C} 23-\mathrm{H} 23$ & 0.9500 \\
\hline $\mathrm{N} 2-\mathrm{C} 38$ & $1.406(3)$ & $\mathrm{C} 24-\mathrm{H} 24 \mathrm{~A}$ & 0.9800 \\
\hline $\mathrm{N} 2-\mathrm{C} 32$ & $1.418(3)$ & $\mathrm{C} 24-\mathrm{H} 24 \mathrm{~B}$ & 0.9800 \\
\hline $\mathrm{C} 1-\mathrm{C} 2$ & $1.425(4)$ & $\mathrm{C} 24-\mathrm{H} 24 \mathrm{C}$ & 0.9800 \\
\hline $\mathrm{C} 1-\mathrm{C} 5$ & $1.432(4)$ & $\mathrm{C} 25-\mathrm{C} 30$ & $1.444(4)$ \\
\hline $\mathrm{C} 1-\mathrm{C} 6$ & $1.500(4)$ & $\mathrm{C} 25-\mathrm{C} 26$ & $1.448(4)$ \\
\hline $\mathrm{C} 2-\mathrm{C} 3$ & $1.421(4)$ & $\mathrm{C} 26-\mathrm{C} 27$ & $1.356(4)$ \\
\hline $\mathrm{C} 2-\mathrm{C} 7$ & $1.503(4)$ & $\mathrm{C} 26-\mathrm{H} 26$ & 0.9500 \\
\hline $\mathrm{C} 3-\mathrm{C} 4$ & $1.422(4)$ & $\mathrm{C} 27-\mathrm{C} 28$ & $1.432(4)$ \\
\hline $\mathrm{C} 3-\mathrm{C} 8$ & $1.500(4)$ & $\mathrm{C} 27-\mathrm{H} 27$ & 0.9500 \\
\hline $\mathrm{C} 4-\mathrm{C} 5$ & $1.414(4)$ & $\mathrm{C} 28-\mathrm{C} 29$ & $1.355(4)$ \\
\hline $\mathrm{C} 4-\mathrm{C} 9$ & $1.499(4)$ & $\mathrm{C} 28-\mathrm{C} 31$ & $1.508(4)$ \\
\hline $\mathrm{C} 5-\mathrm{C} 10$ & $1.501(4)$ & $\mathrm{C} 29-\mathrm{C} 30$ & $1.438(4)$ \\
\hline C6-H6A & 0.9800 & $\mathrm{C} 29-\mathrm{H} 29$ & 0.9500 \\
\hline C6-H6B & 0.9800 & $\mathrm{C} 30-\mathrm{H} 30$ & $1.01(3)$ \\
\hline $\mathrm{C} 6-\mathrm{H} 6 \mathrm{C}$ & 0.9800 & $\mathrm{C} 31-\mathrm{H} 31 \mathrm{~A}$ & 0.9800 \\
\hline $\mathrm{C} 7-\mathrm{H} 7 \mathrm{~A}$ & 0.9800 & $\mathrm{C} 31-\mathrm{H} 31 \mathrm{~B}$ & 0.9800 \\
\hline $\mathrm{C} 7-\mathrm{H} 7 \mathrm{~B}$ & 0.9800 & $\mathrm{C} 31-\mathrm{H} 31 \mathrm{C}$ & 0.9800 \\
\hline $\mathrm{C} 7-\mathrm{H} 7 \mathrm{C}$ & 0.9800 & $\mathrm{C} 32-\mathrm{C} 37$ & $1.399(4)$ \\
\hline $\mathrm{C} 8-\mathrm{H} 8 \mathrm{~A}$ & 0.9800 & $\mathrm{C} 32-\mathrm{C} 33$ & $1.401(4)$ \\
\hline $\mathrm{C} 8-\mathrm{H} 8 \mathrm{~B}$ & 0.9800 & $\mathrm{C} 33-\mathrm{C} 34$ & $1.396(4)$ \\
\hline $\mathrm{C} 8-\mathrm{H} 8 \mathrm{C}$ & 0.9800 & $\mathrm{C} 33-\mathrm{H} 33$ & 0.9500 \\
\hline C9-H9A & 0.9800 & $\mathrm{C} 34-\mathrm{C} 35$ & $1.378(5)$ \\
\hline С9-H9B & 0.9800 & C $34-\mathrm{H} 34$ & 0.9500 \\
\hline
\end{tabular}




\begin{tabular}{|c|c|c|c|}
\hline $\mathrm{C} 9-\mathrm{H} 9 \mathrm{C}$ & 0.9800 & $\mathrm{C} 35-\mathrm{C} 36$ & $1.390(5)$ \\
\hline $\mathrm{C} 10-\mathrm{H} 10 \mathrm{~A}$ & 0.9800 & C $35-\mathrm{H} 35$ & 0.9500 \\
\hline $\mathrm{C} 10-\mathrm{H} 10 \mathrm{~B}$ & 0.9800 & $\mathrm{C} 36-\mathrm{C} 37$ & $1.381(4)$ \\
\hline $\mathrm{C} 10-\mathrm{H} 10 \mathrm{C}$ & 0.9800 & $\mathrm{C} 36-\mathrm{H} 36$ & 0.9500 \\
\hline $\mathrm{C} 11-\mathrm{C} 12$ & $1.394(4)$ & C $37-\mathrm{H} 37$ & 0.9500 \\
\hline $\mathrm{C} 11-\mathrm{C} 16$ & $1.397(4)$ & $\mathrm{C} 38-\mathrm{C} 39$ & $1.399(4)$ \\
\hline $\mathrm{C} 12-\mathrm{C} 13$ & $1.388(4)$ & $\mathrm{C} 38-\mathrm{C} 43$ & $1.411(4)$ \\
\hline $\mathrm{C} 12-\mathrm{H} 12$ & 0.9500 & $\mathrm{C} 39-\mathrm{C} 40$ & $1.392(4)$ \\
\hline $\mathrm{C} 13-\mathrm{C} 14$ & $1.387(5)$ & С $39-\mathrm{H} 39$ & 0.9500 \\
\hline C13-H13 & 0.9500 & $\mathrm{C} 40-\mathrm{C} 41$ & $1.388(4)$ \\
\hline $\mathrm{C} 14-\mathrm{C} 15$ & $1.386(5)$ & $\mathrm{C} 40-\mathrm{H} 40$ & 0.9500 \\
\hline C14-H14 & 0.9500 & $\mathrm{C} 41-\mathrm{C} 42$ & $1.387(4)$ \\
\hline $\mathrm{C} 15-\mathrm{C} 16$ & $1.387(4)$ & $\mathrm{C} 41-\mathrm{H} 41$ & 0.9500 \\
\hline C15-H15 & 0.9500 & $\mathrm{C} 42-\mathrm{C} 43$ & $1.384(4)$ \\
\hline $\mathrm{C} 16-\mathrm{H} 16$ & 0.9500 & $\mathrm{C} 42-\mathrm{H} 42$ & 0.9500 \\
\hline $\mathrm{C} 17-\mathrm{C} 25$ & $1.414(4)$ & $\mathrm{C} 43-\mathrm{H} 43$ & 0.9500 \\
\hline $\mathrm{N} 1-\mathrm{Ti} 1-\mathrm{N} 2$ & $110.42(9)$ & $\mathrm{C} 16-\mathrm{C} 11-\mathrm{N} 1$ & $120.5(2)$ \\
\hline N1-Ti1-C30 & $84.23(9)$ & $\mathrm{C} 13-\mathrm{C} 12-\mathrm{C} 11$ & $120.2(3)$ \\
\hline $\mathrm{N} 2-\mathrm{Ti} 1-\mathrm{C} 30$ & $108.35(9)$ & $\mathrm{C} 13-\mathrm{C} 12-\mathrm{H} 12$ & 119.9 \\
\hline $\mathrm{N} 1-\mathrm{Ti} 1-\mathrm{C} 1$ & $96.36(9)$ & $\mathrm{C} 11-\mathrm{C} 12-\mathrm{H} 12$ & 119.9 \\
\hline $\mathrm{N} 2-\mathrm{Ti} 1-\mathrm{C} 1$ & $110.98(9)$ & $\mathrm{C} 14-\mathrm{C} 13-\mathrm{C} 12$ & $120.2(3)$ \\
\hline $\mathrm{C} 30-\mathrm{Ti} 1-\mathrm{C} 1$ & $137.58(10)$ & $\mathrm{C} 14-\mathrm{C} 13-\mathrm{H} 13$ & 119.9 \\
\hline $\mathrm{N} 1-\mathrm{Ti} 1-\mathrm{C} 2$ & $130.03(9)$ & $\mathrm{C} 12-\mathrm{C} 13-\mathrm{H} 13$ & 119.9 \\
\hline $\mathrm{N} 2-\mathrm{Ti} 1-\mathrm{C} 2$ & $88.09(9)$ & $\mathrm{C} 15-\mathrm{C} 14-\mathrm{C} 13$ & $119.9(3)$ \\
\hline $\mathrm{C} 30-\mathrm{Ti} 1-\mathrm{C} 2$ & $134.76(10)$ & $\mathrm{C} 15-\mathrm{C} 14-\mathrm{H} 14$ & 120.1 \\
\hline $\mathrm{C} 1-\mathrm{T} 11-\mathrm{C} 2$ & $34.80(9)$ & $\mathrm{C} 13-\mathrm{C} 14-\mathrm{H} 14$ & 120.1 \\
\hline $\mathrm{N} 1-\mathrm{Ti1}-\mathrm{C} 5$ & $88.78(9)$ & $\mathrm{C} 14-\mathrm{C} 15-\mathrm{C} 16$ & $120.3(3)$ \\
\hline $\mathrm{N} 2-\mathrm{Ti} 1-\mathrm{C} 5$ & $144.36(9)$ & $\mathrm{C} 14-\mathrm{C} 15-\mathrm{H} 15$ & 119.8 \\
\hline $\mathrm{C} 30-\mathrm{Ti} 1-\mathrm{C} 5$ & $103.08(10)$ & $\mathrm{C} 16-\mathrm{C} 15-\mathrm{H} 15$ & 119.8 \\
\hline $\mathrm{C} 1-\mathrm{Ti} 1-\mathrm{C} 5$ & $34.82(9)$ & $\mathrm{C} 15-\mathrm{C} 16-\mathrm{C} 11$ & $120.0(3)$ \\
\hline $\mathrm{C} 2-\mathrm{Ti} 1-\mathrm{C} 5$ & $57.40(9)$ & $\mathrm{C} 15-\mathrm{C} 16-\mathrm{H} 16$ & 120.0 \\
\hline $\mathrm{N} 1-\mathrm{Ti1}-\mathrm{C} 3$ & $145.52(9)$ & $\mathrm{C} 11-\mathrm{C} 16-\mathrm{H} 16$ & 120.0 \\
\hline $\mathrm{N} 2-\mathrm{Ti} 1-\mathrm{C} 3$ & $100.51(9)$ & $\mathrm{N} 1-\mathrm{C} 17-\mathrm{C} 25$ & $117.0(2)$ \\
\hline $\mathrm{C} 30-\mathrm{Ti} 1-\mathrm{C} 3$ & $100.49(9)$ & $\mathrm{N} 1-\mathrm{C} 17-\mathrm{C} 18$ & $122.0(2)$ \\
\hline $\mathrm{C} 1-\mathrm{Ti1}-\mathrm{C} 3$ & $57.35(9)$ & $\mathrm{C} 25-\mathrm{C} 17-\mathrm{C} 18$ & $120.8(2)$ \\
\hline $\mathrm{C} 2-\mathrm{Ti} 1-\mathrm{C} 3$ & $34.29(9)$ & $\mathrm{N} 1-\mathrm{C} 17-\mathrm{Ti} 1$ & $51.10(12)$ \\
\hline $\mathrm{C} 5-\mathrm{Ti} 1-\mathrm{C} 3$ & $56.80(9)$ & $\mathrm{C} 25-\mathrm{C} 17-\mathrm{Ti} 1$ & $72.43(15)$ \\
\hline $\mathrm{N} 1-\mathrm{Ti1}-\mathrm{C} 4$ & $114.69(9)$ & $\mathrm{C} 18-\mathrm{C} 17-\mathrm{Ti} 1$ & 149.29 (19) \\
\hline $\mathrm{N} 2-\mathrm{Ti} 1-\mathrm{C} 4$ & $134.20(9)$ & $\mathrm{C} 19-\mathrm{C} 18-\mathrm{C} 23$ & $117.8(2)$ \\
\hline $\mathrm{C} 30-\mathrm{Ti} 1-\mathrm{C} 4$ & $83.96(9)$ & $\mathrm{C} 19-\mathrm{C} 18-\mathrm{C} 17$ & $122.3(2)$ \\
\hline $\mathrm{C} 1-\mathrm{Ti} 1-\mathrm{C} 4$ & $57.18(9)$ & $\mathrm{C} 23-\mathrm{C} 18-\mathrm{C} 17$ & $119.9(2)$ \\
\hline $\mathrm{C} 2-\mathrm{Ti} 1-\mathrm{C} 4$ & $56.87(9)$ & $\mathrm{C} 20-\mathrm{C} 19-\mathrm{C} 18$ & $120.8(2)$ \\
\hline $\mathrm{C} 5-\mathrm{Ti} 1-\mathrm{C} 4$ & $33.96(8)$ & $\mathrm{C} 20-\mathrm{C} 19-\mathrm{H} 19$ & 119.6 \\
\hline $\mathrm{C} 3-\mathrm{Ti} 1-\mathrm{C} 4$ & $33.99(9)$ & $\mathrm{C} 18-\mathrm{C} 19-\mathrm{H} 19$ & 119.6 \\
\hline N1-Ti1-C25 & $63.65(9)$ & $\mathrm{C} 19-\mathrm{C} 20-\mathrm{C} 21$ & $121.2(2)$ \\
\hline $\mathrm{N} 2-\mathrm{Ti} 1-\mathrm{C} 25$ & $88.61(9)$ & $\mathrm{C} 19-\mathrm{C} 20-\mathrm{H} 20$ & 119.4 \\
\hline C $30-\mathrm{Ti} 1-\mathrm{C} 25$ & 35.07 (9) & $\mathrm{C} 21-\mathrm{C} 20-\mathrm{H} 20$ & 119.4 \\
\hline
\end{tabular}




$$
\begin{aligned}
& \text { C1-Ti1-C25 } \\
& \text { C2-Ti1-C25 } \\
& \text { C5-Ti1-C25 } \\
& \text { C3-Ti1-C25 } \\
& \text { C4-Ti1-C25 } \\
& \text { N1-Ti1-C17 } \\
& \text { N2-Ti1-C17 } \\
& \text { C30-Ti1-C17 } \\
& \text { C1-Ti1-C17 } \\
& \text { C2-Ti1-C17 } \\
& \text { C5-Ti1-C17 } \\
& \text { C3-Ti1-C17 } \\
& \text { C4-Ti1-C17 } \\
& \text { C25-Ti1-C17 } \\
& \text { C17-N1-C11 } \\
& \text { C17-N1-Ti1 } \\
& \text { C11-N1-Ti1 } \\
& \mathrm{C} 38-\mathrm{N} 2-\mathrm{C} 32 \\
& \text { C38-N2-Ti1 } \\
& \text { C32-N2-Ti1 } \\
& \mathrm{C} 2-\mathrm{C} 1-\mathrm{C} 5 \\
& \mathrm{C} 2-\mathrm{C} 1-\mathrm{C} 6 \\
& \mathrm{C} 5-\mathrm{C} 1-\mathrm{C} 6 \\
& \text { C2-C1-Ti1 } \\
& \text { C5-C1-Ti1 } \\
& \text { C6- C1-Ti1 } \\
& \mathrm{C} 3-\mathrm{C} 2-\mathrm{C} 1 \\
& \mathrm{C} 3-\mathrm{C} 2-\mathrm{C} 7 \\
& \mathrm{C} 1-\mathrm{C} 2-\mathrm{C} 7 \\
& \text { C3-C2-Ti1 } \\
& \mathrm{C} 1-\mathrm{C} 2-\mathrm{Ti} 1 \\
& \text { C7-C2-Ti1 } \\
& \mathrm{C} 2-\mathrm{C} 3-\mathrm{C} 4 \\
& \mathrm{C} 2-\mathrm{C} 3-\mathrm{C} 8 \\
& \mathrm{C} 4-\mathrm{C} 3-\mathrm{C} 8 \\
& \mathrm{C} 2-\mathrm{C} 3-\mathrm{Ti} 1 \\
& \mathrm{C} 4-\mathrm{C} 3-\mathrm{Til} \\
& \text { C8-C3-Til } \\
& \mathrm{C} 5-\mathrm{C} 4-\mathrm{C} 3 \\
& \mathrm{C} 5-\mathrm{C} 4-\mathrm{C} 9 \\
& \mathrm{C} 3-\mathrm{C} 4-\mathrm{C} 9 \\
& \text { C5-C4-Ti1 } \\
& \text { C3-C4-Ti1 } \\
& \text { C9-C4-Ti1 } \\
& \mathrm{C} 4-\mathrm{C} 5-\mathrm{C} 1 \\
& \mathrm{C} 4-\mathrm{C} 5-\mathrm{C} 10 \\
& \mathrm{C} 1-\mathrm{C} 5-\mathrm{C} 10 \\
& \text { C4-C5-Ti1 }
\end{aligned}
$$

156.53 (9)

\begin{tabular}{|c|c|}
\hline $\mathrm{C} 22-\mathrm{C} 21-\mathrm{C} 20$ & $118.1(3)$ \\
\hline $\mathrm{C} 22-\mathrm{C} 21-\mathrm{C} 24$ & $120.5(3)$ \\
\hline $\mathrm{C} 20-\mathrm{C} 21-\mathrm{C} 24$ & $121.5(3)$ \\
\hline $\mathrm{C} 23-\mathrm{C} 22-\mathrm{C} 21$ & $120.9(3)$ \\
\hline $\mathrm{C} 23-\mathrm{C} 22-\mathrm{H} 22$ & 119.5 \\
\hline $\mathrm{C} 21-\mathrm{C} 22-\mathrm{H} 22$ & 119.5 \\
\hline $\mathrm{C} 22-\mathrm{C} 23-\mathrm{C} 18$ & $121.2(3)$ \\
\hline $\mathrm{C} 22-\mathrm{C} 23-\mathrm{H} 23$ & 119.4 \\
\hline $\mathrm{C} 18-\mathrm{C} 23-\mathrm{H} 23$ & 119.4 \\
\hline $\mathrm{C} 21-\mathrm{C} 24-\mathrm{H} 24 \mathrm{~A}$ & 109.5 \\
\hline $\mathrm{C} 21-\mathrm{C} 24-\mathrm{H} 24 \mathrm{~B}$ & 109.5 \\
\hline $\mathrm{H} 24 \mathrm{~A}-\mathrm{C} 24-\mathrm{H} 24 \mathrm{~B}$ & 109.5 \\
\hline $\mathrm{C} 21-\mathrm{C} 24-\mathrm{H} 24 \mathrm{C}$ & 109.5 \\
\hline $\mathrm{H} 24 \mathrm{~A}-\mathrm{C} 24-\mathrm{H} 24 \mathrm{C}$ & 109.5 \\
\hline $\mathrm{H} 24 \mathrm{~B}-\mathrm{C} 24-\mathrm{H} 24 \mathrm{C}$ & 109.5 \\
\hline $\mathrm{C} 17-\mathrm{C} 25-\mathrm{C} 30$ & $122.1(2)$ \\
\hline $\mathrm{C} 17-\mathrm{C} 25-\mathrm{C} 26$ & $121.4(2)$ \\
\hline $\mathrm{C} 30-\mathrm{C} 25-\mathrm{C} 26$ & $116.0(2)$ \\
\hline $\mathrm{C} 17-\mathrm{C} 25-\mathrm{Ti} 1$ & $74.67(15)$ \\
\hline $\mathrm{C} 30-\mathrm{C} 25-\mathrm{Ti} 1$ & $63.98(14)$ \\
\hline $\mathrm{C} 26-\mathrm{C} 25-\mathrm{Ti} 1$ & $129.43(18)$ \\
\hline $\mathrm{C} 27-\mathrm{C} 26-\mathrm{C} 25$ & $120.9(3)$ \\
\hline $\mathrm{C} 27-\mathrm{C} 26-\mathrm{H} 26$ & 119.5 \\
\hline $\mathrm{C} 25-\mathrm{C} 26-\mathrm{H} 26$ & 119.5 \\
\hline $\mathrm{C} 26-\mathrm{C} 27-\mathrm{C} 28$ & $122.3(3)$ \\
\hline $\mathrm{C} 26-\mathrm{C} 27-\mathrm{H} 27$ & 118.9 \\
\hline $\mathrm{C} 28-\mathrm{C} 27-\mathrm{H} 27$ & 118.9 \\
\hline $\mathrm{C} 29-\mathrm{C} 28-\mathrm{C} 27$ & $118.3(3)$ \\
\hline $\mathrm{C} 29-\mathrm{C} 28-\mathrm{C} 31$ & $122.6(3)$ \\
\hline $\mathrm{C} 27-\mathrm{C} 28-\mathrm{C} 31$ & $119.1(3)$ \\
\hline $\mathrm{C} 28-\mathrm{C} 29-\mathrm{C} 30$ & $121.7(3)$ \\
\hline $\mathrm{C} 28-\mathrm{C} 29-\mathrm{H} 29$ & 119.1 \\
\hline $\mathrm{C} 30-\mathrm{C} 29-\mathrm{H} 29$ & 119.1 \\
\hline $\mathrm{C} 29-\mathrm{C} 30-\mathrm{C} 25$ & $119.3(2)$ \\
\hline $\mathrm{C} 29-\mathrm{C} 30-\mathrm{Ti} 1$ & $135.2(2)$ \\
\hline $\mathrm{C} 25-\mathrm{C} 30-\mathrm{Ti} 1$ & $80.95(16)$ \\
\hline $\mathrm{C} 29-\mathrm{C} 30-\mathrm{H} 30$ & $113.2(19)$ \\
\hline $\mathrm{C} 25-\mathrm{C} 30-\mathrm{H} 30$ & $119.2(19)$ \\
\hline $\mathrm{Ti} 1-\mathrm{C} 30-\mathrm{H} 30$ & 82.8 (19) \\
\hline $\mathrm{C} 28-\mathrm{C} 31-\mathrm{H} 31 \mathrm{~A}$ & 109.5 \\
\hline $\mathrm{C} 28-\mathrm{C} 31-\mathrm{H} 31 \mathrm{~B}$ & 109.5 \\
\hline $\mathrm{H} 31 \mathrm{~A}-\mathrm{C} 31-\mathrm{H} 31 \mathrm{~B}$ & 109.5 \\
\hline $\mathrm{C} 28-\mathrm{C} 31-\mathrm{H} 31 \mathrm{C}$ & 109.5 \\
\hline $\mathrm{H} 31 \mathrm{~A}-\mathrm{C} 31-\mathrm{H} 31 \mathrm{C}$ & 109.5 \\
\hline $\mathrm{H} 31 \mathrm{~B}-\mathrm{C} 31-\mathrm{H} 31 \mathrm{C}$ & 109.5 \\
\hline $\mathrm{C} 37-\mathrm{C} 32-\mathrm{C} 33$ & $118.7(3)$ \\
\hline $\mathrm{C} 37-\mathrm{C} 32-\mathrm{N} 2$ & $120.2(2)$ \\
\hline $\mathrm{C} 33-\mathrm{C} 32-\mathrm{N} 2$ & $121.1(2)$ \\
\hline
\end{tabular}

166.07 (9)

$127.03(9)$

$133.74(9)$

117.99 (8)

$33.24(9)$

$92.07(9)$

$63.01(9)$

$129.47(9)$

$160.82(9)$

$117.37(9)$

$162.00(9)$

$130.97(9)$

$32.90(8)$

$119.2(2)$

$95.66(16)$

145.04 (18)

$114.7(2)$

$126.47(17)$

$118.83(16)$

$107.4(2)$

$125.2(2)$

$126.0(2)$

$72.94(15)$

73.64 (14)

$129.66(19)$

$108.4(2)$

$126.1(2)$

$125.3(3)$

$74.53(15)$

$72.26(15)$

$123.27(19)$

107.7 (2)

$126.1(2)$

$125.4(2)$

71.17 (14)

$73.17(14)$

$128.83(18)$

108.4 (2)

$125.6(2)$

$125.3(2)$

$71.88(14)$

72.85 (14)

128.42 (18)

108.1 (2)

125.1 (3)

126.4 (2)

74.15 (14)

\author{
118.1 (3) \\ 121.5 (3) \\ 120.9 (3) \\ 119.5 \\ 121.2 (3) \\ 119.4 \\ 119.4 \\ 109.5 \\ 109.5 \\ 122.1 (2) \\ $121.4(2)$ \\ 116.0 (2) \\ 63.98 (14) \\ $129.43(18)$ \\ 119.5 \\ 119.5 \\ 12.3 (3) \\ 118.9 \\ 118.3 (3) \\ $122.6(3)$ \\ 119.1 (3) \\ 121.7 (3) \\ 119.1 \\ 119.1 \\ 119.3 (2) \\ 135.2 (2) \\ $113.2(19)$ \\ 119.2 (19) \\ 82.8 (19) \\ 109.5 \\ 109.5 \\ 109.5 \\ 109.5 \\ 118.7 (3) \\ $121.1(2)$
}




\begin{tabular}{|c|c|c|c|}
\hline $\mathrm{C} 1-\mathrm{C} 5-\mathrm{Ti} 1$ & $71.54(14)$ & $\mathrm{C} 34-\mathrm{C} 33-\mathrm{C} 32$ & $119.9(3)$ \\
\hline $\mathrm{C} 10-\mathrm{C} 5-\mathrm{Ti} 1$ & $125.75(18)$ & $\mathrm{C} 34-\mathrm{C} 33-\mathrm{H} 33$ & 120.1 \\
\hline $\mathrm{C} 1-\mathrm{C} 6-\mathrm{H} 6 \mathrm{~A}$ & 109.5 & $\mathrm{C} 32-\mathrm{C} 33-\mathrm{H} 33$ & 120.1 \\
\hline $\mathrm{C} 1-\mathrm{C} 6-\mathrm{H} 6 \mathrm{~B}$ & 109.5 & $\mathrm{C} 35-\mathrm{C} 34-\mathrm{C} 33$ & $120.9(3)$ \\
\hline $\mathrm{H} 6 \mathrm{~A}-\mathrm{C} 6-\mathrm{H} 6 \mathrm{~B}$ & 109.5 & $\mathrm{C} 35-\mathrm{C} 34-\mathrm{H} 34$ & 119.5 \\
\hline $\mathrm{C} 1-\mathrm{C} 6-\mathrm{H} 6 \mathrm{C}$ & 109.5 & $\mathrm{C} 33-\mathrm{C} 34-\mathrm{H} 34$ & 119.5 \\
\hline $\mathrm{H} 6 \mathrm{~A}-\mathrm{C} 6-\mathrm{H} 6 \mathrm{C}$ & 109.5 & $\mathrm{C} 34-\mathrm{C} 35-\mathrm{C} 36$ & $119.2(3)$ \\
\hline $\mathrm{H} 6 \mathrm{~B}-\mathrm{C} 6-\mathrm{H} 6 \mathrm{C}$ & 109.5 & $\mathrm{C} 34-\mathrm{C} 35-\mathrm{H} 35$ & 120.4 \\
\hline $\mathrm{C} 2-\mathrm{C} 7-\mathrm{H} 7 \mathrm{~A}$ & 109.5 & $\mathrm{C} 36-\mathrm{C} 35-\mathrm{H} 35$ & 120.4 \\
\hline $\mathrm{C} 2-\mathrm{C} 7-\mathrm{H} 7 \mathrm{~B}$ & 109.5 & $\mathrm{C} 37-\mathrm{C} 36-\mathrm{C} 35$ & $120.7(3)$ \\
\hline $\mathrm{H} 7 \mathrm{~A}-\mathrm{C} 7-\mathrm{H} 7 \mathrm{~B}$ & 109.5 & $\mathrm{C} 37-\mathrm{C} 36-\mathrm{H} 36$ & 119.7 \\
\hline $\mathrm{C} 2-\mathrm{C} 7-\mathrm{H} 7 \mathrm{C}$ & 109.5 & $\mathrm{C} 35-\mathrm{C} 36-\mathrm{H} 36$ & 119.7 \\
\hline $\mathrm{H} 7 \mathrm{~A}-\mathrm{C} 7-\mathrm{H} 7 \mathrm{C}$ & 109.5 & $\mathrm{C} 36-\mathrm{C} 37-\mathrm{C} 32$ & $120.6(3)$ \\
\hline $\mathrm{H} 7 \mathrm{~B}-\mathrm{C} 7-\mathrm{H} 7 \mathrm{C}$ & 109.5 & $\mathrm{C} 36-\mathrm{C} 37-\mathrm{H} 37$ & 119.7 \\
\hline $\mathrm{C} 3-\mathrm{C} 8-\mathrm{H} 8 \mathrm{~A}$ & 109.5 & $\mathrm{C} 32-\mathrm{C} 37-\mathrm{H} 37$ & 119.7 \\
\hline $\mathrm{C} 3-\mathrm{C} 8-\mathrm{H} 8 \mathrm{~B}$ & 109.5 & $\mathrm{C} 39-\mathrm{C} 38-\mathrm{N} 2$ & $120.0(2)$ \\
\hline $\mathrm{H} 8 \mathrm{~A}-\mathrm{C} 8-\mathrm{H} 8 \mathrm{~B}$ & 109.5 & $\mathrm{C} 39-\mathrm{C} 38-\mathrm{C} 43$ & $118.1(2)$ \\
\hline $\mathrm{C} 3-\mathrm{C} 8-\mathrm{H} 8 \mathrm{C}$ & 109.5 & $\mathrm{~N} 2-\mathrm{C} 38-\mathrm{C} 43$ & $121.9(2)$ \\
\hline $\mathrm{H} 8 \mathrm{~A}-\mathrm{C} 8-\mathrm{H} 8 \mathrm{C}$ & 109.5 & $\mathrm{C} 40-\mathrm{C} 39-\mathrm{C} 38$ & $120.5(2)$ \\
\hline $\mathrm{H} 8 \mathrm{~B}-\mathrm{C} 8-\mathrm{H} 8 \mathrm{C}$ & 109.5 & $\mathrm{C} 40-\mathrm{C} 39-\mathrm{H} 39$ & 119.7 \\
\hline $\mathrm{C} 4-\mathrm{C} 9-\mathrm{H} 9 \mathrm{~A}$ & 109.5 & $\mathrm{C} 38-\mathrm{C} 39-\mathrm{H} 39$ & 119.7 \\
\hline $\mathrm{C} 4-\mathrm{C} 9-\mathrm{H} 9 \mathrm{~B}$ & 109.5 & $\mathrm{C} 41-\mathrm{C} 40-\mathrm{C} 39$ & $120.8(3)$ \\
\hline $\mathrm{H} 9 \mathrm{~A}-\mathrm{C} 9-\mathrm{H} 9 \mathrm{~B}$ & 109.5 & $\mathrm{C} 41-\mathrm{C} 40-\mathrm{H} 40$ & 119.6 \\
\hline $\mathrm{C} 4-\mathrm{C} 9-\mathrm{H} 9 \mathrm{C}$ & 109.5 & $\mathrm{C} 39-\mathrm{C} 40-\mathrm{H} 40$ & 119.6 \\
\hline $\mathrm{H} 9 \mathrm{~A}-\mathrm{C} 9-\mathrm{H} 9 \mathrm{C}$ & 109.5 & $\mathrm{C} 42-\mathrm{C} 41-\mathrm{C} 40$ & $119.1(3)$ \\
\hline $\mathrm{H} 9 \mathrm{~B}-\mathrm{C} 9-\mathrm{H} 9 \mathrm{C}$ & 109.5 & $\mathrm{C} 42-\mathrm{C} 41-\mathrm{H} 41$ & 120.4 \\
\hline $\mathrm{C} 5-\mathrm{C} 10-\mathrm{H} 10 \mathrm{~A}$ & 109.5 & $\mathrm{C} 40-\mathrm{C} 41-\mathrm{H} 41$ & 120.4 \\
\hline $\mathrm{C} 5-\mathrm{C} 10-\mathrm{H} 10 \mathrm{~B}$ & 109.5 & $\mathrm{C} 43-\mathrm{C} 42-\mathrm{C} 41$ & $120.9(3)$ \\
\hline $\mathrm{H} 10 \mathrm{~A}-\mathrm{C} 10-\mathrm{H} 10 \mathrm{~B}$ & 109.5 & $\mathrm{C} 43-\mathrm{C} 42-\mathrm{H} 42$ & 119.6 \\
\hline $\mathrm{C} 5-\mathrm{C} 10-\mathrm{H} 10 \mathrm{C}$ & 109.5 & $\mathrm{C} 41-\mathrm{C} 42-\mathrm{H} 42$ & 119.6 \\
\hline $\mathrm{H} 10 \mathrm{~A}-\mathrm{C} 10-\mathrm{H} 10 \mathrm{C}$ & 109.5 & $\mathrm{C} 42-\mathrm{C} 43-\mathrm{C} 38$ & $120.6(3)$ \\
\hline $\mathrm{H} 10 \mathrm{~B}-\mathrm{C} 10-\mathrm{H} 10 \mathrm{C}$ & 109.5 & $\mathrm{C} 42-\mathrm{C} 43-\mathrm{H} 43$ & 119.7 \\
\hline $\mathrm{C} 12-\mathrm{C} 11-\mathrm{C} 16$ & $119.3(2)$ & $\mathrm{C} 38-\mathrm{C} 43-\mathrm{H} 43$ & 119.7 \\
\hline $\mathrm{C} 12-\mathrm{C} 11-\mathrm{N} 1$ & $120.2(2)$ & & \\
\hline $\mathrm{C} 5-\mathrm{C} 1-\mathrm{C} 2-\mathrm{C} 3$ & $-0.2(3)$ & $\mathrm{N} 1-\mathrm{C} 17-\mathrm{C} 18-\mathrm{C} 23$ & $-142.0(3)$ \\
\hline $\mathrm{C} 6-\mathrm{C} 1-\mathrm{C} 2-\mathrm{C} 3$ & $166.8(3)$ & $\mathrm{C} 25-\mathrm{C} 17-\mathrm{C} 18-\mathrm{C} 23$ & $43.1(4)$ \\
\hline $\mathrm{Ti} 1-\mathrm{C} 1-\mathrm{C} 2-\mathrm{C} 3$ & $-66.34(18)$ & $\mathrm{Ti} 1-\mathrm{C} 17-\mathrm{C} 18-\mathrm{C} 23$ & $151.4(3)$ \\
\hline $\mathrm{C} 5-\mathrm{C} 1-\mathrm{C} 2-\mathrm{C} 7$ & $-175.2(2)$ & $\mathrm{C} 23-\mathrm{C} 18-\mathrm{C} 19-\mathrm{C} 20$ & $-2.1(4)$ \\
\hline $\mathrm{C} 6-\mathrm{C} 1-\mathrm{C} 2-\mathrm{C} 7$ & $-8.3(4)$ & $\mathrm{C} 17-\mathrm{C} 18-\mathrm{C} 19-\mathrm{C} 20$ & $177.2(3)$ \\
\hline $\mathrm{T} 11-\mathrm{C} 1-\mathrm{C} 2-\mathrm{C} 7$ & $118.6(3)$ & $\mathrm{C} 18-\mathrm{C} 19-\mathrm{C} 20-\mathrm{C} 21$ & $-0.1(4)$ \\
\hline $\mathrm{C} 5-\mathrm{C} 1-\mathrm{C} 2-\mathrm{Ti} 1$ & $66.13(17)$ & $\mathrm{C} 19-\mathrm{C} 20-\mathrm{C} 21-\mathrm{C} 22$ & $2.1(4)$ \\
\hline $\mathrm{C} 6-\mathrm{C} 1-\mathrm{C} 2-\mathrm{Ti} 1$ & $-126.9(3)$ & $\mathrm{C} 19-\mathrm{C} 20-\mathrm{C} 21-\mathrm{C} 24$ & $-176.4(3)$ \\
\hline $\mathrm{C} 1-\mathrm{C} 2-\mathrm{C} 3-\mathrm{C} 4$ & $0.3(3)$ & $\mathrm{C} 20-\mathrm{C} 21-\mathrm{C} 22-\mathrm{C} 23$ & $-1.8(4)$ \\
\hline $\mathrm{C} 7-\mathrm{C} 2-\mathrm{C} 3-\mathrm{C} 4$ & $175.3(2)$ & $\mathrm{C} 24-\mathrm{C} 21-\mathrm{C} 22-\mathrm{C} 23$ & $176.7(3)$ \\
\hline $\mathrm{Ti} 1-\mathrm{C} 2-\mathrm{C} 3-\mathrm{C} 4$ & $-64.51(17)$ & $\mathrm{C} 21-\mathrm{C} 22-\mathrm{C} 23-\mathrm{C} 18$ & $-0.5(4)$ \\
\hline $\mathrm{C} 1-\mathrm{C} 2-\mathrm{C} 3-\mathrm{C} 8$ & $-170.3(2)$ & $\mathrm{C} 19-\mathrm{C} 18-\mathrm{C} 23-\mathrm{C} 22$ & $2.4(4)$ \\
\hline $\mathrm{C} 7-\mathrm{C} 2-\mathrm{C} 3-\mathrm{C} 8$ & $4.7(4)$ & $\mathrm{C} 17-\mathrm{C} 18-\mathrm{C} 23-\mathrm{C} 22$ & $-176.9(3)$ \\
\hline
\end{tabular}




$\begin{array}{ll}\text { Ti1-C2-C3-C8 } & 124.8(3) \\ \text { C1-C2-C3-Ti1 } & 64.84(18) \\ \text { C7-C2-C3-Ti1 } & -120.2(3) \\ \text { C2-C3-C4-C5 } & -0.3(3) \\ \text { C8-C3-C4-C5 } & 170.4(2) \\ \text { Ti1-C3-C4-C5 } & -63.53(17) \\ \text { C2-C3-C4-C9 } & -171.5(2) \\ \text { C8-C3-C4-C9 } & -0.7(4) \\ \text { Ti1-C3-C4-C9 } & 125.3(3) \\ \text { C2-C3-C4-Ti1 } & 63.20(18) \\ \text { C8-C3-C4-Ti1 } & -126.1(2) \\ \text { C3-C4-C5-C1 } & 0.2(3) \\ \text { C9-C4-C5-C1 } & 171.3(2) \\ \text { Ti1-C4-C5-C1 } & -63.95(17) \\ \text { C3-C4-C5-C10 } & -173.0(2) \\ \text { C9-C4-C5-C10 } & -1.9(4) \\ \text { Ti1-C4-C5-C10 } & 122.9(3) \\ \text { C3-C4-C5-Ti1 } & 64.16(17) \\ \text { C9-C4-C5-Ti1 } & -124.8(3) \\ \text { C2-C1-C5-C4 } & 0.0(3) \\ \text { C6-C1-C5-C4 } & -166.8(3) \\ \text { Ti1-C1-C5-C4 } & 65.66(17) \\ \text { C2-C1-C5-C10 } & 173.1(2) \\ \text { C6-C1-C5-C10 } & 6.2(4) \\ \text { Ti1-C1-C5-C10 } & -121.3(3) \\ \text { C2-C1-C5-Ti1 } & -65.66(18) \\ \text { C6-C1-C5-Ti1 } & 127.5(3) \\ \text { C17-N1-C11-C12 } & 65.8(3) \\ \text { Ti1-N1-C11-C12 } & -119.8(3) \\ \text { C17-N1-C11-C16 } & -114.5(3) \\ \text { Ti1-N1-C11-C16 } & 59.9(4) \\ \text { C16-C11-C12-C13 } & -1.0(4) \\ \text { N1-C11-C12-C13 } & 178.6(2) \\ \text { C11-C12-C13-C14 } & 1.5(4) \\ \text { C12-C13-C14-C15 } & -0.5(4) \\ \text { C13-C14-C15-C16 } & -0.9(4) \\ \text { C14-C15-C16-C11 } & 1.3(4) \\ \text { C12-C11-C16-C15 } & -0.4(4) \\ \text { N1-C11-C16-C15 } & 180.0(2) \\ \text { C11-N1-C17-C25 } & -151.1(2) \\ \text { Ti1-N1-C17-C25 } & 32.1(2) \\ \text { C11-N1-C17-C18 } & 33.8(4) \\ \text { Ti1-N1-C17-C18 } & -143.0(2) \\ \text { C11-N1-C17-Ti1 } & 176.8(3) \\ \text { N1-C17-C18-C19 } & 38.7(4) \\ \text { C25-C17-C18-C19 } & -136.2(3) \\ \text { Ti1-C17-C18-C19 } & -27.9(5) \\ & \end{array}$

\begin{tabular}{|c|c|}
\hline $\mathrm{N} 1-\mathrm{C} 17-\mathrm{C} 25-\mathrm{C} 30$ & $19.1(4)$ \\
\hline $\mathrm{C} 18-\mathrm{C} 17-\mathrm{C} 25-\mathrm{C} 30$ & $-165.8(2)$ \\
\hline $\mathrm{Ti} 1-\mathrm{C} 17-\mathrm{C} 25-\mathrm{C} 30$ & $44.8(2)$ \\
\hline $\mathrm{N} 1-\mathrm{C} 17-\mathrm{C} 25-\mathrm{C} 26$ & $-152.9(2)$ \\
\hline $\mathrm{C} 18-\mathrm{C} 17-\mathrm{C} 25-\mathrm{C} 26$ & $22.3(4)$ \\
\hline $\mathrm{Ti} 1-\mathrm{C} 17-\mathrm{C} 25-\mathrm{C} 26$ & $-127.2(2)$ \\
\hline $\mathrm{N} 1-\mathrm{C} 17-\mathrm{C} 25-\mathrm{Ti} 1$ & $-25.7(2)$ \\
\hline $\mathrm{C} 18-\mathrm{C} 17-\mathrm{C} 25-\mathrm{Ti} 1$ & $149.4(2)$ \\
\hline $\mathrm{C} 17-\mathrm{C} 25-\mathrm{C} 26-\mathrm{C} 27$ & $-177.9(3)$ \\
\hline $\mathrm{C} 30-\mathrm{C} 25-\mathrm{C} 26-\mathrm{C} 27$ & $9.6(4)$ \\
\hline $\mathrm{Ti} 1-\mathrm{C} 25-\mathrm{C} 26-\mathrm{C} 27$ & $86.3(3)$ \\
\hline $\mathrm{C} 25-\mathrm{C} 26-\mathrm{C} 27-\mathrm{C} 28$ & $0.1(4)$ \\
\hline $\mathrm{C} 26-\mathrm{C} 27-\mathrm{C} 28-\mathrm{C} 29$ & $-5.7(4)$ \\
\hline $\mathrm{C} 26-\mathrm{C} 27-\mathrm{C} 28-\mathrm{C} 31$ & $173.3(3)$ \\
\hline $\mathrm{C} 27-\mathrm{C} 28-\mathrm{C} 29-\mathrm{C} 30$ & $0.9(4)$ \\
\hline $\mathrm{C} 31-\mathrm{C} 28-\mathrm{C} 29-\mathrm{C} 30$ & $-178.1(3)$ \\
\hline $\mathrm{C} 28-\mathrm{C} 29-\mathrm{C} 30-\mathrm{C} 25$ & $9.2(4)$ \\
\hline $\mathrm{C} 28-\mathrm{C} 29-\mathrm{C} 30-\mathrm{Ti} 1$ & $-98.8(3)$ \\
\hline $\mathrm{C} 17-\mathrm{C} 25-\mathrm{C} 30-\mathrm{C} 29$ & $173.6(2)$ \\
\hline $\mathrm{C} 26-\mathrm{C} 25-\mathrm{C} 30-\mathrm{C} 29$ & $-14.1(4)$ \\
\hline $\mathrm{Ti} 1-\mathrm{C} 25-\mathrm{C} 30-\mathrm{C} 29$ & $-137.3(3)$ \\
\hline $\mathrm{C} 17-\mathrm{C} 25-\mathrm{C} 30-\mathrm{Ti} 1$ & $-49.1(2)$ \\
\hline $\mathrm{C} 26-\mathrm{C} 25-\mathrm{C} 30-\mathrm{Ti} 1$ & $123.2(2)$ \\
\hline $\mathrm{C} 38-\mathrm{N} 2-\mathrm{C} 32-\mathrm{C} 37$ & $55.4(3)$ \\
\hline $\mathrm{Ti} 1-\mathrm{N} 2-\mathrm{C} 32-\mathrm{C} 37$ & $-122.8(2)$ \\
\hline $\mathrm{C} 38-\mathrm{N} 2-\mathrm{C} 32-\mathrm{C} 33$ & $-124.6(3)$ \\
\hline $\mathrm{Ti} 1-\mathrm{N} 2-\mathrm{C} 32-\mathrm{C} 33$ & $57.2(3)$ \\
\hline $\mathrm{C} 37-\mathrm{C} 32-\mathrm{C} 33-\mathrm{C} 34$ & $0.6(4)$ \\
\hline $\mathrm{N} 2-\mathrm{C} 32-\mathrm{C} 33-\mathrm{C} 34$ & $-179.4(2)$ \\
\hline $\mathrm{C} 32-\mathrm{C} 33-\mathrm{C} 34-\mathrm{C} 35$ & $-0.5(4)$ \\
\hline $\mathrm{C} 33-\mathrm{C} 34-\mathrm{C} 35-\mathrm{C} 36$ & $0.4(4)$ \\
\hline $\mathrm{C} 34-\mathrm{C} 35-\mathrm{C} 36-\mathrm{C} 37$ & $-0.3(5)$ \\
\hline $\mathrm{C} 35-\mathrm{C} 36-\mathrm{C} 37-\mathrm{C} 32$ & $0.3(5)$ \\
\hline $\mathrm{C} 33-\mathrm{C} 32-\mathrm{C} 37-\mathrm{C} 36$ & $-0.5(4)$ \\
\hline $\mathrm{N} 2-\mathrm{C} 32-\mathrm{C} 37-\mathrm{C} 36$ & $179.5(3)$ \\
\hline $\mathrm{C} 32-\mathrm{N} 2-\mathrm{C} 38-\mathrm{C} 39$ & $-148.6(2)$ \\
\hline Ti1 $-\mathrm{N} 2-\mathrm{C} 38-\mathrm{C} 39$ & $29.5(3)$ \\
\hline $\mathrm{C} 32-\mathrm{N} 2-\mathrm{C} 38-\mathrm{C} 43$ & $32.7(3)$ \\
\hline $\mathrm{Ti} 1-\mathrm{N} 2-\mathrm{C} 38-\mathrm{C} 43$ & $-149.2(2)$ \\
\hline $\mathrm{N} 2-\mathrm{C} 38-\mathrm{C} 39-\mathrm{C} 40$ & $-176.5(2)$ \\
\hline $\mathrm{C} 43-\mathrm{C} 38-\mathrm{C} 39-\mathrm{C} 40$ & $2.3(4)$ \\
\hline $\mathrm{C} 38-\mathrm{C} 39-\mathrm{C} 40-\mathrm{C} 41$ & $-1.7(4)$ \\
\hline $\mathrm{C} 39-\mathrm{C} 40-\mathrm{C} 41-\mathrm{C} 42$ & $-0.3(4)$ \\
\hline $\mathrm{C} 40-\mathrm{C} 41-\mathrm{C} 42-\mathrm{C} 43$ & $1.8(4)$ \\
\hline $\mathrm{C} 41-\mathrm{C} 42-\mathrm{C} 43-\mathrm{C} 38$ & $-1.2(4)$ \\
\hline $\mathrm{C} 39-\mathrm{C} 38-\mathrm{C} 43-\mathrm{C} 42$ & $-0.8(4)$ \\
\hline $\mathrm{N} 2-\mathrm{C} 38-\mathrm{C} 43-\mathrm{C} 42$ & $177.9(2)$ \\
\hline
\end{tabular}

